\title{
Stabilization of a Strained Heteroradialene by Peripheral Electron Delocalization
}

\author{
S. Hessam M. Mehr, Brian O. Patrick, and Mark J. MacLachlan
}

Department of Chemistry, University of British Columbia, 2036 Main Mall, Vancouver, BC V6T 1Z1 CANADA

mmaclach@chem.ubc.ca

$\begin{array}{ll}\text { Materials } & \text { S2 }\end{array}$

$\begin{array}{ll}\text { Instrumentation } & \mathrm{S} 2\end{array}$

X-ray Crystallography $\quad$ S2

Cyclic Voltammetry $\quad$ S2

NMR Spectroscopy $\quad$ S3

Experimental $\quad$ S3

$\begin{array}{ll}\text { NMR Spectra } & \text { S6 }\end{array}$

$\begin{array}{ll}\mathrm{Ab} \text { initio calculations } & \mathrm{S} 9\end{array}$

Geometry Optimization $\quad$ S9

Conformational Analysis of Compound $3 \quad$ S9

Calculation of Excited-State Energies $\quad \mathrm{S} 10$

Calculated Energy-Optimized Atomic Coordinates for 3 (S1) S11

Calculated Energy-Optimized Atomic Coordinates for S2 $\quad \mathrm{S} 12$

Calculated Energy-Optimized Atomic Coordinates for S3 $\quad$ S13

Calculated Energy-Optimized Atomic Coordinates for S4 $\quad$ S14

$\begin{array}{ll}\text { References } & \text { S15 }\end{array}$ 


\section{Materials}

All reagents and solvents were obtained from standard suppliers and used without purification. 1,3-Diformylphloroglucinol was prepared from phloroglucinol in $85 \%$ yield by following the literature procedure. ${ }^{15} \mathrm{~N}$-labelled dimethylamine hydrochloride was obtained from Berry \& Associates.

\section{Instrumentation}

All reactions were carried out under air unless otherwise noted. High-resolution ESI mass spectra were obtained on a Waters/Micromass LCT time-of-flight (TOF) mass spectrometer. Infrared spectroscopy was carried out on a Thermo Scientific Nicolet 6700 (ATR) FT-IR instrument using the SmartOrbit attenuated total reflectance (ATR) accessory.

\section{X-ray Crystallography}

Slow evaporation of a 1:2 $\mathrm{CH}_{2} \mathrm{Cl}_{2}$-hexanes solution of compound $\mathbf{3}$ gave crystals suitable for single-crystal X-ray crystallography.

All measurements were made at a temperature of $-183.0 \pm 0.1{ }^{\circ} \mathrm{C}$ on a Bruker APEX DUO diffractometer using graphite monochromated Mo-Ka $\left(\max 2 \theta=53.0^{\circ}\right)$. Data was collected in a series of $\phi$ and $\omega$ scans in $0.5^{\circ}$ oscillations using 15.0-second exposures. The crystal-to-detector distance was $38.21 \mathrm{~mm}$. The material crystallizes as a two-component 'split-crystal' with components one and two related by a $180^{\circ}$ rotation about the $(0.000 .001 .00)$ recirpocal axis. Data were integrated for both components, including both overlapped and non-overlapped reflections. In total 13148 reflections were integrated (2245 from component one only, 2234 from component two only, 8669 overlapped). Data were collected and integrated using the Bruker SAINT software packages. Data were corrected for absorption effects using the multiscan technique (TWINABS), with minimum and maximum transmission coefficients of 0.851 and 0.991, respectively. The data were corrected for Lorentz and polarization effects.

The structure was solved by direct methods $^{2}$ using non-overlapped data from the major twin component. Subsequent refinements were carried out using an HKLF 5 format data set containing complete data from component one and overlapped reflections from component two. All non-hydrogen atoms were refined anisotropically. All hydrogen atoms were placed in calculated positions. The final cycle of full-matrix least-squares refinement on $\mathrm{F}^{2}$ was based on 2944 reflections and 197 variable parameters.

X-ray crystal data for $3: \mathrm{C}_{15} \mathrm{H}_{21} \mathrm{~N}_{3} \mathrm{O}_{3}, \mathrm{MW}=291.35 \mathrm{~g} \mathrm{~mol}^{-1}$, colorless irregular crystal $(0.10$ $\left.\times 0.17 \times 0.28 \mathrm{~mm}^{3}\right)$, triclinic, space group P-1 (\#2), a = 6.433(2), b = 7.542(3), c = 15.771(6) $\mathrm{A}$, $\alpha=90.874(6)^{\circ}, \beta=95.675(7)^{\circ}, \gamma=109.593(8)^{\circ}, \mathrm{V}=716.5(4) \AA^{3}, Z=2, \rho_{\text {calcd }}=1.351 \mathrm{~g} \mathrm{~cm}^{-3}$, $\mathrm{F}_{000}=312.00, \mu(\mathrm{Mo}-\mathrm{K} \alpha)=0.95 \mathrm{~cm}^{-1}, \lambda(\mathrm{Mo}-\mathrm{K} \alpha)=0.71073 \AA$, GoF $=1.05 ; \mathrm{R} 1, \mathrm{wR} 2=0.065$, 0.144 (all data); 0.052, $0.137\left(\mathrm{~F}^{2}: \mathrm{I}>2.00 \sigma(\mathrm{I})\right)$.

\section{Cyclic Voltammetry}

Cyclic voltammetry experiments were carried out on a Pine Instruments AFCBP1 potentiostat. The setup comprised dichloromethane as solvent, tetrabutylammonium hexafluorophosphate as electrolyte, a platinum mesh counter electrode, a glassy carbon working electrode, silver wire used as pseudoreference, and ferrocene (added after the measurement) as reference. 


\section{NMR Spectroscopy}

All ${ }^{1} \mathrm{H}$ NMR and ${ }^{1} \mathrm{H}-{ }^{15} \mathrm{~N}$ HSQC spectra were measured on a $400 \mathrm{MHz}$ Bruker spectrometer equipped with an inverse (proton detection) probe.

Direct observation of heteronuclei was accomplished on a $400 \mathrm{MHz}$ Bruker spectrometer configured with a SmartProbe ${ }^{\mathrm{TM}}$ using a flip angle of $30^{\circ}$ and inverse-gated $\left({ }^{15} \mathrm{~N}\right)$ or power-gated $\left({ }^{13} \mathrm{C}\right)$ proton decoupling. To speed up longitudinal relaxation, about $4 \mathrm{mg}$ of $\mathrm{Cr}(\mathrm{acac})_{3}$ was added. This relaxation agent was excluded in ${ }^{1} \mathrm{H}$ experiments to prevent signal broadening. The ${ }^{15} \mathrm{~N}$ spectral reference was adjusted to the Bruker scale (liquid $\mathrm{NH}_{3}$ at $0 \mathrm{ppm}$ ). A standard sample of $90 \%$ formamide in DMSO- $d_{6}$ was used as indirect reference to verify this.

For ${ }^{1} \mathrm{H}-{ }^{15} \mathrm{~N}$ HSQC a phase-sensitive pulse sequence incorporating gradient pulses and double INEPT transfer was used (HSQCETGP). ${ }^{3,4} \mathrm{~A}$ wide ${ }^{15} \mathrm{~N}$ chemical shift window (250 ppm) was used initially and a $30 \mathrm{ppm}$ area containing all ${ }^{15} \mathrm{~N}-{ }^{1} \mathrm{H}$ cross peaks was selected for subsequent measurements. Direct (see above) and indirect chemical shifts for ${ }^{15} \mathrm{~N}$ agreed within the heteronuclear resolution of the HSQC experiment $(24 \mathrm{~Hz})$ in all cases.

All NMR experiments were carried out using Bruker TopSpin ${ }^{\mathrm{TM}}$ software and the data subsequently processed using ACD/Labs NMR Processor. For 1D heteronuclear spectra, an exponential window function with a line-broadening factor (LB) of 3.0 was employed.

Rotational activation barriers were calculated from the coalescence temperatures obtained in variable-temperature NMR experiments. The common definition ${ }^{5}$ of coalescence, $\mathrm{k}_{\mathrm{ex}}=\frac{\pi \Delta v}{\sqrt{2}}$ was used. The Eyring equation, $\mathrm{k}_{\mathrm{ex}}=\frac{\mathrm{k}_{\mathrm{B}} \mathrm{T}}{\mathrm{h}} e^{\frac{-\Delta \mathrm{G}^{\ddagger}}{R T}}$ then yields

$$
\Delta \mathrm{G}^{\ddagger}=-R T_{c} \ln \frac{\pi h \Delta v}{\sqrt{2} k_{B} T_{c}} .
$$

The uncertainty in $\Delta \mathrm{G}^{\ddagger}$ was calculated based on an assumed $T_{c}$ error of $\pm 5^{\circ} \mathrm{C}$ and

$$
\frac{\partial \Delta G^{\ddagger}}{\partial T_{c}}=\frac{\Delta G^{\ddagger}}{T_{c}}+\mathrm{R} .
$$

\section{Experimental}

The following improved procedure was used to prepare 2,4,6-triformylphloroglucinol 1 from 2,4-diformylphloroglucinol (DFP). DFP itself was prepared from phloroglucinol in $85 \%$ yield by following the literature procedure. ${ }^{1}$

Scheme S1. Improved synthesis of 1.<smiles>Oc1cc(O)cc(O)c1</smiles>

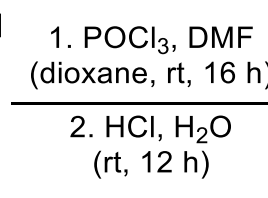<smiles>O=Cc1c(O)cc(O)c(C=O)c1O</smiles>

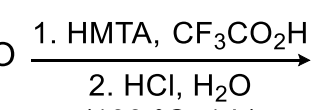
$\left(100^{\circ} \mathrm{C}, 1 \mathrm{~h}\right)$<smiles>O=Cc1c(O)c(C=O)c(O)c(C=O)c1O</smiles>

Synthesis of triformylphloroglucinol (1): Diformylphloroglucinol (6.00 g, $32.9 \mathrm{mmol}, 1$ equiv) and hexamethylenetetramine (6.96 g, $49.6 \mathrm{mmol}, 1.5 \mathrm{equiv})$ were dried under high vacuum in a Schlenk flask at $60{ }^{\circ} \mathrm{C}$ for $1.5 \mathrm{~h}$. The flask was back-filled with $\mathrm{N}_{2}$ and trifluoroacetic acid (38.0 mL, $496 \mathrm{mmol}, 15$ equiv) was added. Following $4 \mathrm{~h}$ of reacting at 
$100{ }^{\circ} \mathrm{C}$ under a nitrogen atmosphere, $2 \mathrm{M} \mathrm{HCl}(120 \mathrm{~mL})$ was added and stirring continued under air at the same temperature for $1 \mathrm{~h}$. During this period a large amount of orange precipitate formed, which was subsequently removed by filtration through celite. After cooling to room temperature, the supernatant was extracted with $3 \times 50 \mathrm{~mL}$ of dichloromethane and the organic extract dried over anhydrous $\mathrm{Na}_{2} \mathrm{SO}_{4}$, filtered, and evaporated to give $4.12 \mathrm{~g}$ (19.6 mmol, 60\%) of compound $\mathbf{1}$ as a salmon-orange powder. This is a substantial improvement on the previously reported syntheses of $\mathbf{1}$.

\section{Scheme S2. Synthesis of 3}

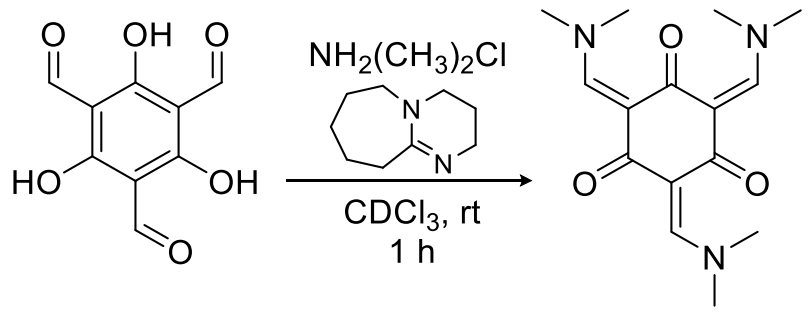

Synthesis of 3: Triformylphloroglucinol $1(10.0 \mathrm{mg}, 0.0476 \mathrm{mmol}, 1$ equiv) was added to a septum-capped NMR tube containing $0.6 \mathrm{~mL}$ of $\mathrm{CDCl}_{3}$ and dispersed by sonicating for $5 \mathrm{~min}$. A septum-capped Schlenk flask was charged with $50 \mathrm{mg}$ (0.61 mmol, 13 equiv) of anhydrous dimethylamine hydrochloride and $0.100 \mathrm{~mL}$ (101 mg, $0.67 \mathrm{mmol}, 14$ equiv) of 1,8diazabicycloundec-7-ene (DBU) under nitrogen and sonicated until a viscous, white suspension was obtained. Dimethylamine was carried from the headspace of the Schlenk flask by nitrogen gas introduced through the sidearm and, using a steel canula, bubbled into the NMR solvent. After $1 \mathrm{~min}$, the Schlenk flask was brought in contact with a heating bath at $95{ }^{\circ} \mathrm{C}$, causing bubbles of dimethylamine gas to form in the suspension. During the following $20 \mathrm{~min}$, the suspension of 1 in $\mathrm{CDCl}_{3}$ underwent a change of color from the initial pale yellow to a brilliant fluorescent yellow-green, to a bright orange.

At this point, the cannula was removed and the progress of the reaction was monitored by ${ }^{1} \mathrm{H}$ NMR spectroscopy. The reaction was complete after about $1 \mathrm{~h}$, judging by the disappearance of the aldehyde peak. Evaporation of NMR solvent under high vacuum left $13.7 \mathrm{mg}(0.0471 \mathrm{mmol}$, $99 \%$ ) of an orange powder. ${ }^{1} \mathrm{H}$ NMR $\left(400 \mathrm{MHz}, \mathrm{CD}_{3} \mathrm{OD}\right) \delta 8.10(\mathrm{~s}, 3 \mathrm{H} ; \mathrm{CH}), 3.43\left(\mathrm{~s}, 9 \mathrm{H} ; \mathrm{CH}_{3}\right)$, $3.19\left(\mathrm{~s}, 9 \mathrm{H} ; \mathrm{CH}_{3}\right) .{ }^{13} \mathrm{C} \mathrm{NMR}\left(100 \mathrm{MHz}, \mathrm{CDCl}_{3}\right) \delta 182.7(\mathrm{CO}$, br), $159.5(\mathrm{CH}-\mathrm{N}), 107.4(C-$ $\mathrm{CHN}$, br), $48.2\left(\mathrm{CH}_{3}\right), 44.4\left(\mathrm{CH}_{3}\right) .{ }^{15} \mathrm{~N}$ NMR $\left(\mathbf{3 -}^{15} \mathrm{~N}_{3}, 40 \mathrm{MHz}, \mathrm{CD}_{3} \mathrm{OD}\right) \delta$ 130.2. UV-Vis $\left(\mathrm{CH}_{2} \mathrm{Cl}_{2}\right) \lambda_{\max }(\varepsilon)=363\left(5.3 \times 10^{4}\right), 308\left(4.1 \times 10^{4}\right), 257\left(1.3 \times 10^{4}\right) \mathrm{nm}\left(\mathrm{cm}^{-1} \mathrm{~mol}^{-1} \mathrm{~L}\right)$. IR (neat) $v=2918$ (br), 2806, 1582, 1525, 1414, 1339, 1293, 1135, 1042, 998, 967, 944, 809, 733, 625, 588, $463 \mathrm{~cm}^{-1}$. HRMS (ESI/TOF-Q) $\mathrm{m} / z:[3+\mathrm{H}]^{+}$Calcd for $\mathrm{C}_{15} \mathrm{H}_{22} \mathrm{~N}_{3} \mathrm{O}_{3}$ 292.1661; Found 292.1663.

Synthesis of 3- ${ }^{15} \mathbf{N}_{3}$ : The preparation of $3-{ }^{15} \mathbf{N}_{3}$ was carried out identically to that of 3 except using ${ }^{15} \mathrm{~N}$-labelled anhydrous dimethylamine hydrochloride instead of anhydrous dimethylamine hydrochloride. IR (neat) $v=2917$ (br), 2806, 1573, 1520, 1411, 1344, 1290, 1134, 1038, 997, 945, 810, 733, 624, 586, $461 \mathrm{~cm}^{-1}$. HRMS (ESI/TOF-Q) $\mathrm{m} / z:\left[\mathbf{3}^{15} \mathbf{N}_{\mathbf{3}}+\mathrm{H}\right]^{+}$Calc'd for $\mathrm{C}_{15} \mathrm{H}_{22}{ }^{15} \mathrm{~N}_{3} \mathrm{O}_{3}$ 295.1572; Found 295.1567 (base peak); Calcd for $\mathrm{C}_{15} \mathrm{H}_{22}{ }^{15} \mathrm{~N}_{2} \mathrm{NO}_{3} 294.1602$; Found $294.1581(6.5 \%)$. 


\section{Scheme S3. Synthesis of 4}<smiles>CCN(C=C1C(=O)C(=CN(CC)CC)C(=O)C(=CN(CC)CC)C1=O)CC</smiles>

Synthesis of 4: Triformylphloroglucinol 1 (10.0 mg, $0.0476 \mathrm{mmol}, 1$ equiv) was introduced to a septum-capped NMR tube containing $0.6 \mathrm{~mL}$ of $\mathrm{CD}_{3} \mathrm{OD}$; the contents were degassed with $\mathrm{N}_{2}$. Diethylamine $(0.10 \mathrm{~mL}, 71 \mathrm{mg}, 0.97 \mathrm{mmol}, 20$ equiv) was injected into a Schlenk flask and the flask warmed up to $40{ }^{\circ} \mathrm{C}$ to accelerate the evaporation of the amine while the gaseous diethylamine was bubbled into the NMR solvent through a canula. During the next 15 min the solids of $\mathbf{1}$ dissolved to give a yellow-orange solution. Following this, the canula was removed and the NMR tube stored at room temperature for $16 \mathrm{~h}$. The ${ }^{1} \mathrm{H}$ NMR spectrum of the resulting orange-red solution shows virtually complete conversion of TFP to $\mathbf{4}$, along with a large excess of diethylamine. Solvent and excess diethylamine were then removed at room temperature under high vacuum, leaving a dark crimson semi-solid. When dissolved in benzene, a light orange supernatant and small amounts of a sticky dark solid resulted. Evaporation of the supernatant under high vacuum gave a thick orange oil $(17.7 \mathrm{mg}, 0.0467 \mathrm{mmol}, 98 \%)$ which is pure 4 by ${ }^{1} \mathrm{H}$ NMR spectroscopy. ${ }^{1} \mathrm{H}$ NMR $\left(400 \mathrm{MHz}, \mathrm{CDCl}_{3}\right) \delta 8.10(\mathrm{~s}, 3 \mathrm{H} ; \mathrm{CH}), 3.88\left(\mathrm{q}, \mathrm{br},{ }^{3} J_{\mathrm{HH}}=7.04 \mathrm{~Hz}\right.$, $\left.6 \mathrm{H} ; \mathrm{CH}_{2}\right), 3.52\left(\mathrm{q}, \mathrm{br},{ }^{3} J_{\mathrm{HH}}=7.04 \mathrm{~Hz}, 6 \mathrm{H} ; \mathrm{CH}_{2}\right), 1.33\left(\mathrm{t}, \mathrm{br},{ }^{3} J_{\mathrm{HH}}=7.04 \mathrm{~Hz}, 9 \mathrm{H} ; \mathrm{CH}_{3}\right), 1.16(\mathrm{t}$, br, $\left.{ }^{3} J_{\mathrm{HH}}=7.04 \mathrm{~Hz}, 9 \mathrm{H} ; \mathrm{CH}_{3}\right) .{ }^{13} \mathrm{C} \mathrm{NMR}\left(100 \mathrm{MHz}, \mathrm{CDCl}_{3}\right) \delta 183.1(\mathrm{CO}), 157.2(\mathrm{CH}-\mathrm{N}), 107.4$ $(C-\mathrm{CHN}), 53.5\left(\mathrm{CH}_{2}\right), 47.8\left(\mathrm{CH}_{2}\right), 15.2\left(\mathrm{CH}_{3}\right), 12.7\left(\mathrm{CH}_{3}\right)$. UV-Vis $\left(\mathrm{CH}_{2} \mathrm{Cl}_{2}\right) \lambda_{\max }(\varepsilon)=365(3.6$

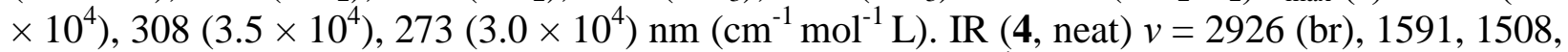
1414, 1371, 1138, 1043, 1003, 947, 810, 627, 590, $469 \mathrm{~cm}^{-1}$. IR (neat glaze) $v=2973,2934$, 2873, 1573, 1515, 1442, 1389, 1346, 1301, 1259, 1178, 1138, 1049, 1020, 938, 924, 807, 619, 591, 529, $476 \mathrm{~cm}^{-1}$. HRMS (ESI/TOF-Q) $\mathrm{m} / z$ : $[4+\mathrm{H}]^{+}$Calcd for $\mathrm{C}_{21} \mathrm{H}_{34} \mathrm{~N}_{3} \mathrm{O}_{3} 376.2600$; Found 376.2595 . 


\section{NMR Spectra}

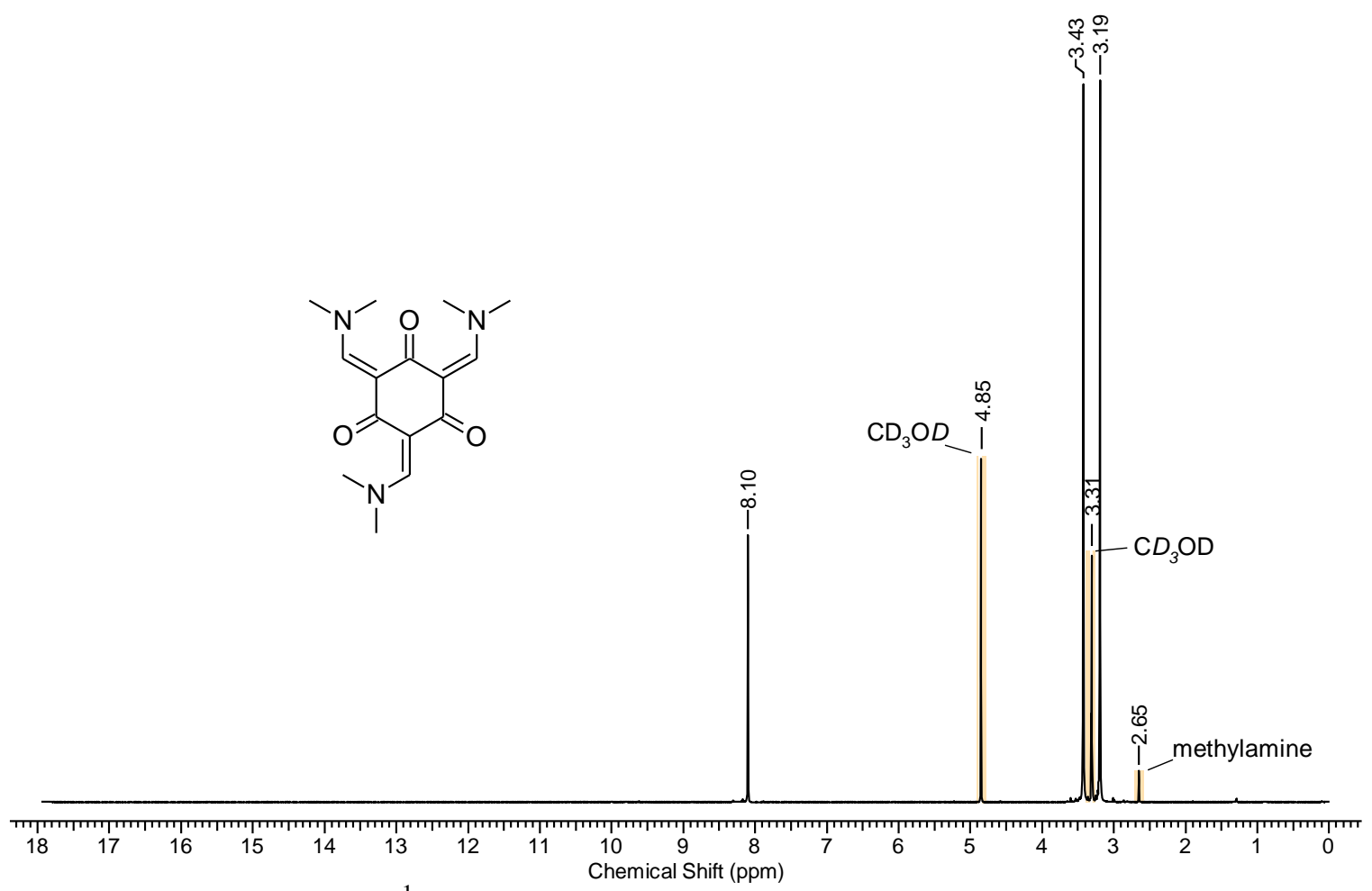

Figure S1. ${ }^{1} \mathrm{H}$ NMR spectrum of $\mathbf{3}\left(400 \mathrm{MHz}, \mathrm{CD}_{3} \mathrm{OD}\right)$.

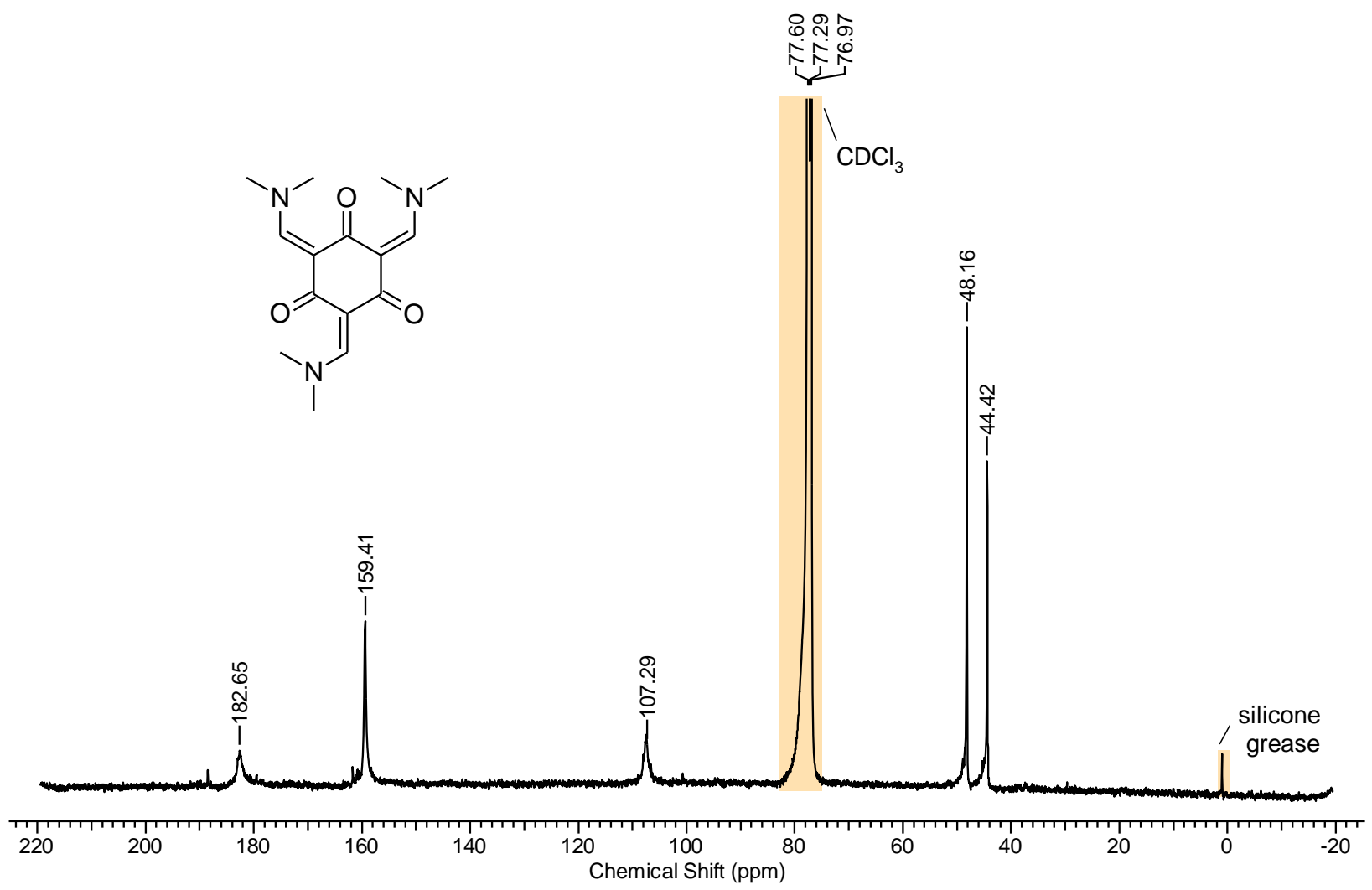

Figure S2. ${ }^{13} \mathrm{C}$ NMR spectrum of $\mathbf{3}\left(100 \mathrm{MHz}, \mathrm{CDCl}_{3}\right)$. 


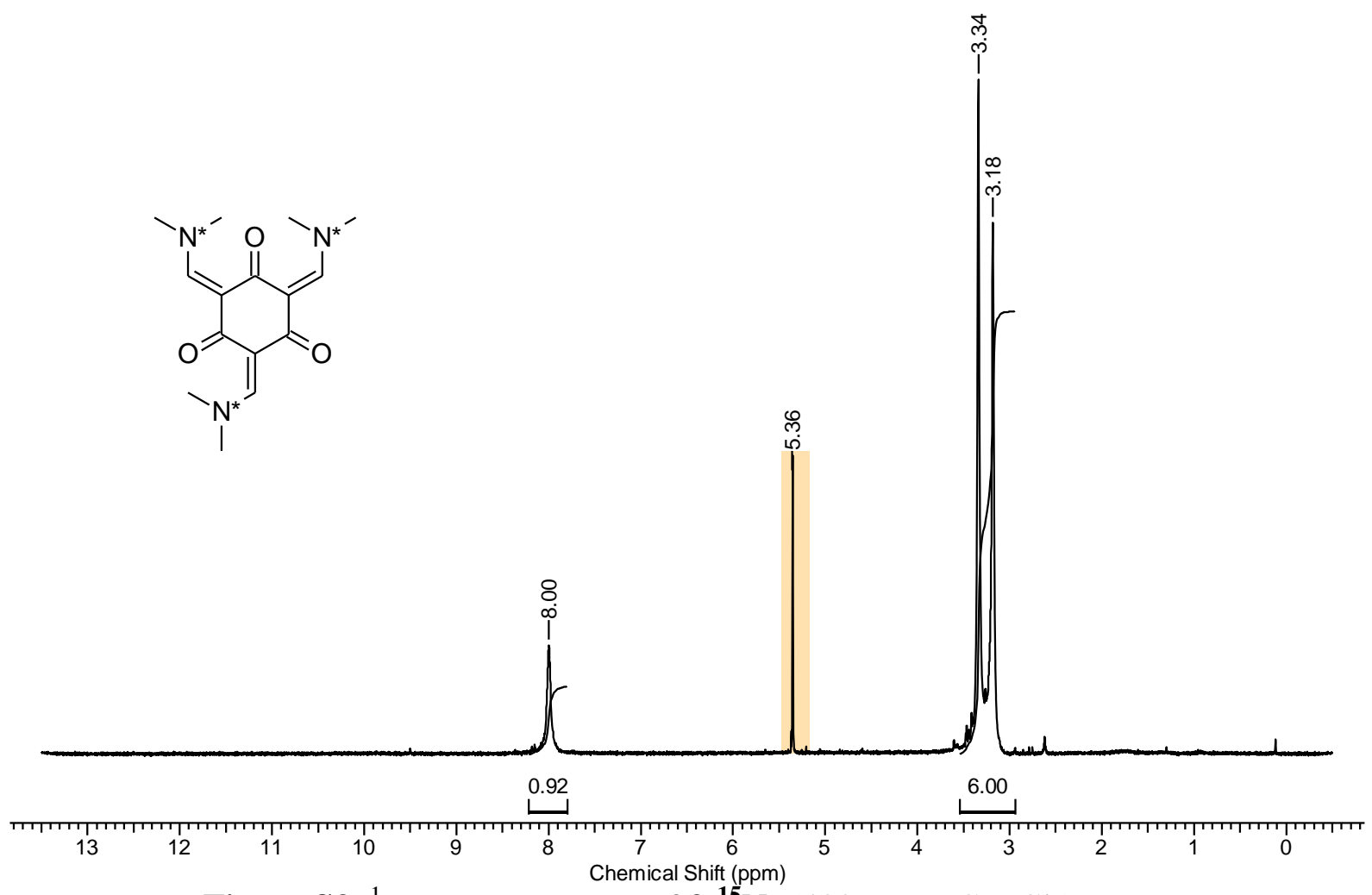

Figure S3. ${ }^{1} \mathrm{H}$ NMR spectrum of $\mathbf{3 -}^{\mathbf{1 5}} \mathbf{N}_{\mathbf{3}}\left(400 \mathrm{MHz}, \mathrm{CD}_{2} \mathrm{Cl}_{2}\right)$.

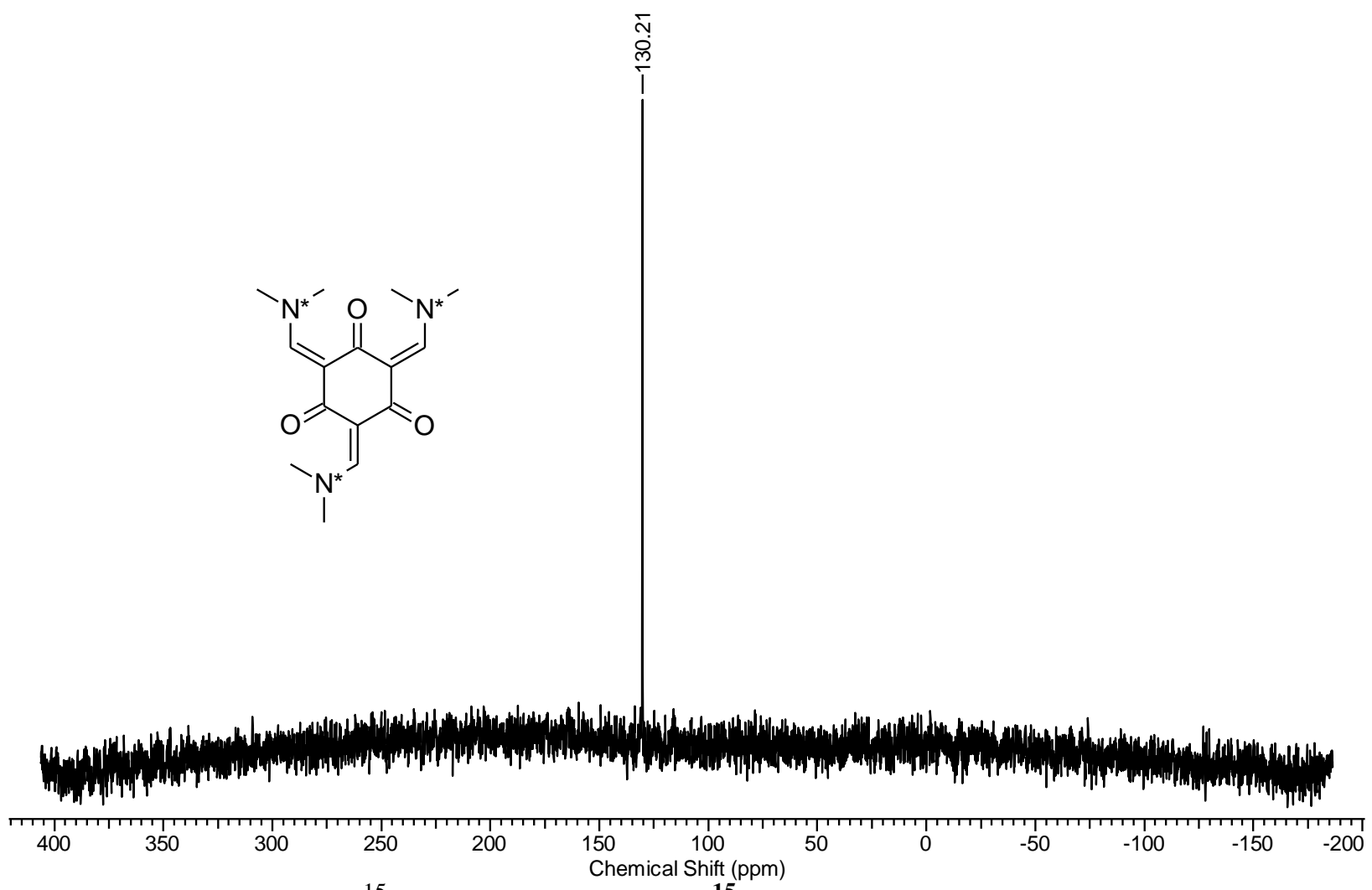

Figure S4. ${ }^{15} \mathrm{~N}$ NMR spectrum of $\mathbf{3 -}^{\mathbf{1 5}} \mathbf{N}_{\mathbf{3}}\left(40 \mathrm{MHz}, \mathrm{CD}_{3} \mathrm{OD}\right)$. 


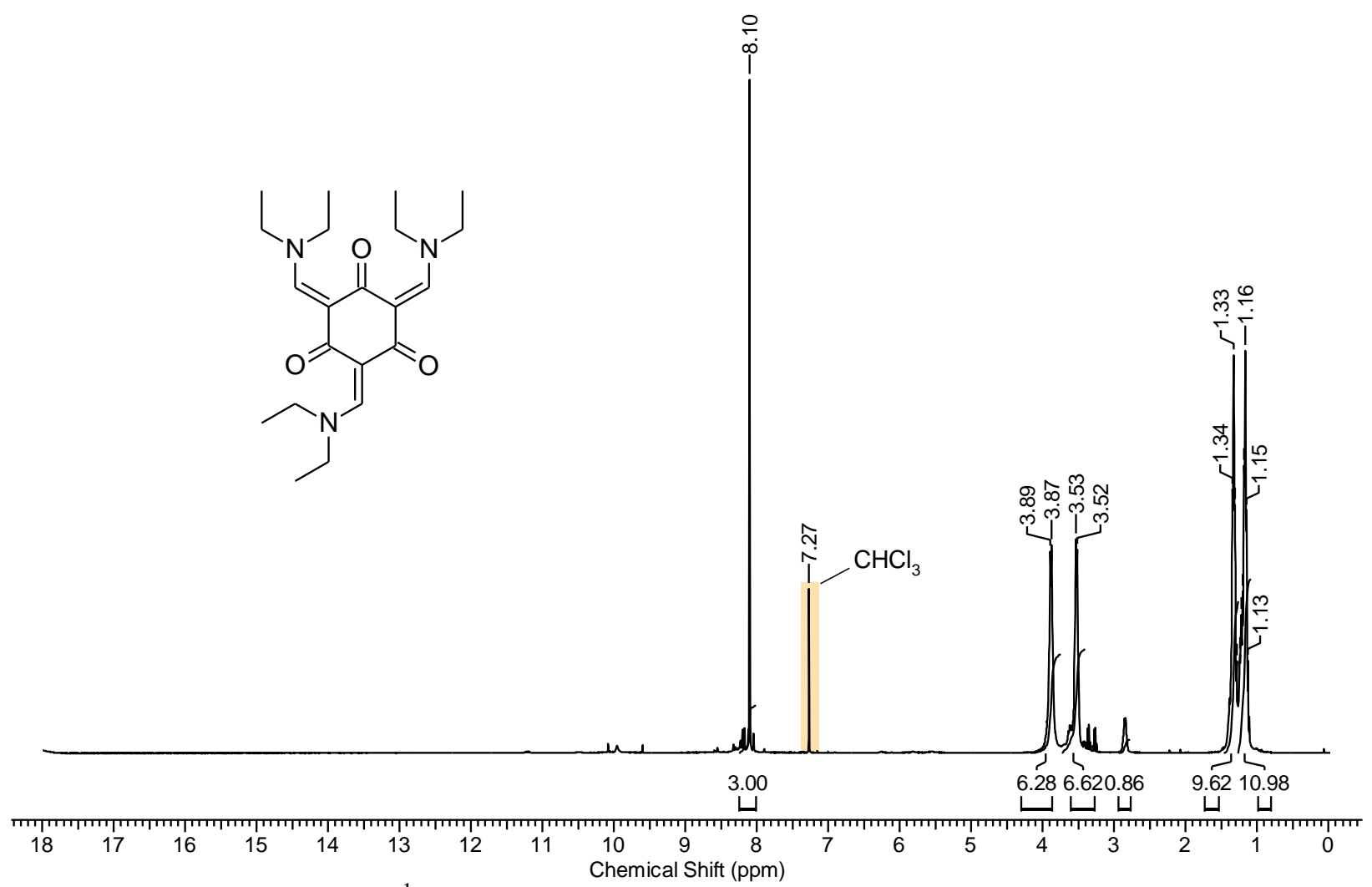

Figure S5. ${ }^{1} \mathrm{H}$ NMR spectrum of $4\left(400 \mathrm{MHz}, \mathrm{CDCl}_{3}\right)$.

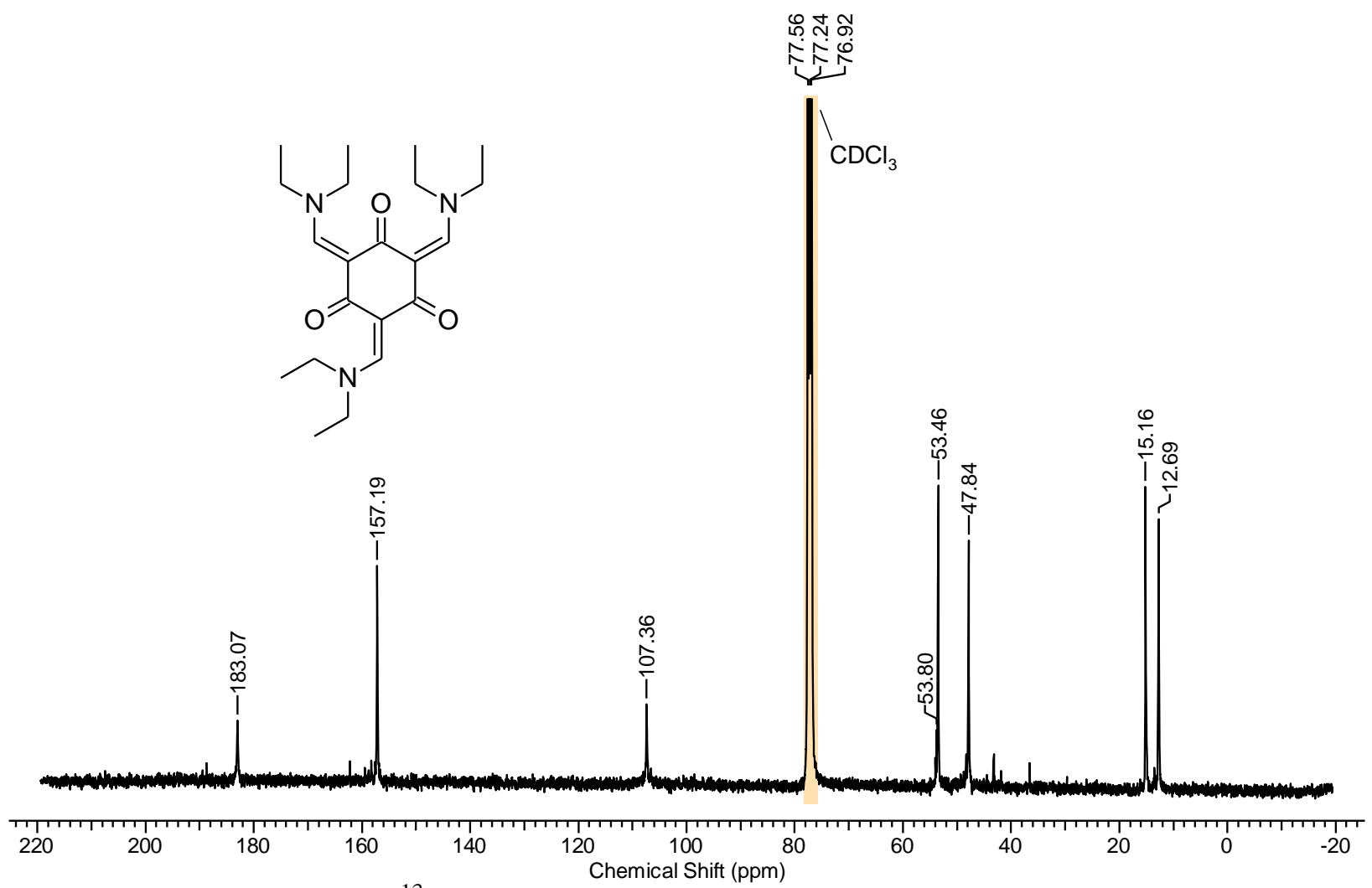

Figure S6. ${ }^{13} \mathrm{C}$ NMR spectrum of $\mathbf{4}\left(100 \mathrm{MHz}, \mathrm{CDCl}_{3}\right)$. 


\section{Ab initio calculations}

All ab initio calculations were carried out using Gaussian $09^{6}$. Specifically, density functional theory (DFT) was used with the Minnesota $06^{7}$ (M06) functional and a Dunning-type triple zeta correlation consistent basis set (cc-pVTZ) ${ }^{8-10}$. In all cases, the polarizable continuum model (PCM) ${ }^{11}$ was been used to model the effects of bulk solvent. For geometry optimizations, the final structure was checked for the absence of imaginary frequencies.

\section{Geometry Optimization}

The atomic coordinates obtained from single-crystal X-ray diffraction of $\mathbf{3}$ were subjected to energy optimization in bulk $\mathrm{CH}_{2} \mathrm{Cl}_{2}$. Figure $\mathrm{S} 7$ shows the initial and final geometry along with the evolution of the $\mathrm{O}^{1} \mathrm{C}^{11} \mathrm{C}^{12} \mathrm{C}^{15}$ and $\mathrm{O}^{3} \mathrm{C}^{18} \mathrm{C}^{10} \mathrm{C}^{15}$ dihedral angles. The former is relaxed by less than $2^{\circ}$ while the latter's deviation from planarity becomes more pronounced.
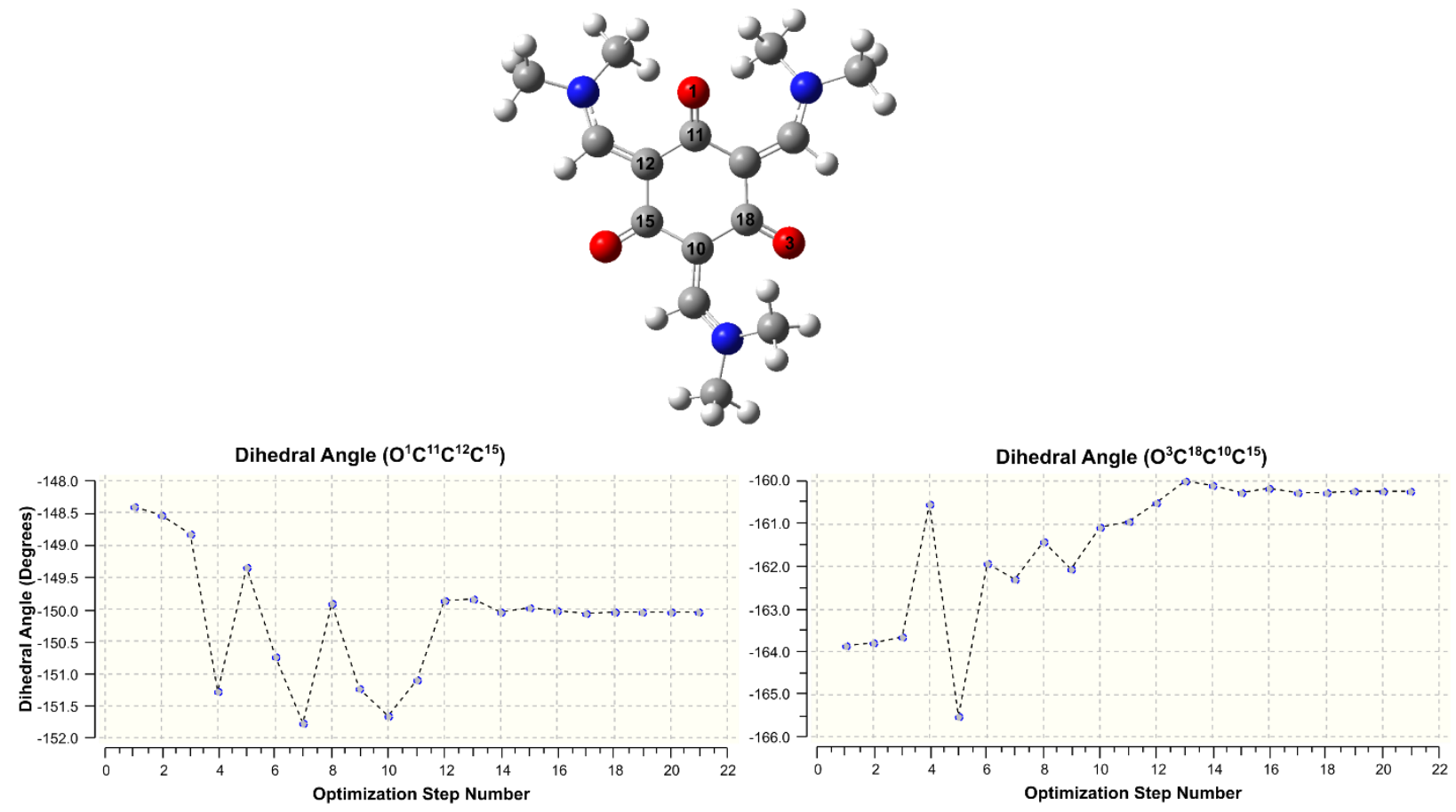

Figure S7: Energy optimized geometry for 3 (top) and the evolution of the $\mathrm{O}^{1} \mathrm{C}^{11} \mathrm{C}^{12} \mathrm{C}^{15}$ (bottom left) and $\mathrm{O}^{3} \mathrm{C}^{18} \mathrm{C}^{10} \mathrm{C}^{15}$ (bottom right) dihedral angles.

\section{Conformational Analysis of Compound 3}

NMR experiments at low temperature revealed an asymmetric conformer of $\mathbf{3}$ as the majority species. Does the conformation in this maximally stable conformer resemble that seen in the solid state? To answer this question, we manually constructed and optimized the geometry

of a number of alternative conformations of $\mathbf{3}$ with different orientations of the $=\mathrm{CH}-\mathrm{N}\left(\mathrm{CH}_{3}\right)_{2}$ arms. 
<smiles>CN(C)C[C@H]1C(O)[C@H](CN(C)C)[C@@H](O)[C@H](CN(C)C)[C@H]1O</smiles>

s1

(seen in SCXRD) $0.0 \mathrm{kcal} / \mathrm{mol}$<smiles>CN(C)CC1C(O)[C@H](CN(C)C)[C@@H](O)C(CN(C)C)[C@@H]1O</smiles>

s2<smiles>CN(C)C[C@H]1C(O)C(C[NH+](C)C)[C@@H](O)[C@H](CN(C)C)[C@H]1O</smiles>

S3

$0.8 \mathrm{kcal} / \mathrm{mol}$<smiles>CNCC1[C@H](O)C(CN(C)C)C(O)C(CN(C)C)[C@H]1O</smiles>

S4

$1.7 \mathrm{kcal} / \mathrm{mol}$

Figure S8. Relative energy values for optimized candidate geometries of 3 relative to $S 1$.

As Figure S8 shows, starting with the geometry seen in the solid state leads to the lowestenergy isomer (S1) among the examined candidates. However, given the small energy difference and the limitations of the PCM model, one can only speculate that $\mathbf{S 1}$ is the dominant structure seen in our low-temperature NMR experiments.

\section{Calculation of Excited-State Energies}

Time-dependent density functional theory ${ }^{12-15}$ was used to search for the lowest-lying singlet excited states. Table S1 summarizes the results the transitions with oscillator strength > 0.1 . For each excitation, the contribution of each major Slater determinant is presented as $100 \%$ $\times 2 x^{2}$ where $x$ is the coefficient for the determinant in the configuration interaction (CI) expansion in the Gaussian output.

Table S1. Electronic excited states of 3.

\begin{tabular}{|c|c|c|c|c|c|}
\hline \multirow{2}{*}{ Energy (eV) } & \multirow{2}{*}{ Wavelength (nm) } & \multirow{2}{*}{ Oscillator strength } & \multicolumn{3}{|c|}{ Contributors } \\
\hline & & & from & to & $\%$ \\
\hline \multirow{2}{*}{3.5462} & \multirow{2}{*}{349.63} & \multirow{2}{*}{0.1410} & HOMO & LUMO & 79 \\
\hline & & & HOMO-2 & LUMO & 17 \\
\hline \multirow{5}{*}{3.7774} & \multirow{5}{*}{328.23} & \multirow{5}{*}{0.1169} & HOMO-5 & LUMO+2 & 2 \\
\hline & & & HOMO-4 & LUMO+1 & 4 \\
\hline & & & HOMO-2 & LUMO & 66 \\
\hline & & & HOMO-1 & LUMO & 6 \\
\hline & & & HOMO & LUMO & 18 \\
\hline \multirow{2}{*}{3.8124} & \multirow{2}{*}{325.21} & \multirow{2}{*}{0.4751} & HOMO-2 & LUMO & 4 \\
\hline & & & HOMO-1 & LUMO & 92 \\
\hline \multirow{5}{*}{4.3405} & \multirow{5}{*}{285.64} & \multirow{5}{*}{0.4001} & HOMO-5 & LUMO & 3 \\
\hline & & & HOMO-3 & LUMO & 10 \\
\hline & & & HOMO-1 & LUMO+1 & 36 \\
\hline & & & HOMO-1 & LUMO+2 & 8 \\
\hline & & & HOMO & $\mathrm{LUMO}+2$ & 37 \\
\hline \multirow{3}{*}{4.4028} & \multirow{3}{*}{281.60} & \multirow{3}{*}{0.3076} & HOMO-3 & LUMO & 9 \\
\hline & & & HOMO-1 & LUMO+1 & 22 \\
\hline & & & HOMO-1 & $\mathrm{LUMO}+2$ & 53 \\
\hline
\end{tabular}




\begin{tabular}{|c|c|c|c|c|c|}
\hline \multirow{5}{*}{4.4218} & \multirow{5}{*}{280.39} & \multirow{5}{*}{0.1440} & HOMO & LUMO+1 & 11 \\
\hline & & & HOMO-3 & LUMO & 57 \\
\hline & & & HOMO-1 & LUMO+1 & 11 \\
\hline & & & HOMO-1 & LUMO+2 & 21 \\
\hline & & & HOMO & LUMO+2 & 5 \\
\hline
\end{tabular}

Calculated Energy-Optimized Atomic Coordinates for 3 (S1)

The atomic coordinates for $\mathbf{3}$ obtained from single-crystal X-ray diffraction were subjected to energy optimization in bulk (PCM) $\mathrm{CH}_{2} \mathrm{Cl}_{2}$. The following listing shows the Cartesian coordinates of the optimized structure in angstroms.

\begin{tabular}{|c|c|c|c|}
\hline 0 & -2.08466700 & 0.38131600 & 1.08307700 \\
\hline 0 & 0.95641700 & -2.82736700 & -0.32154800 \\
\hline 0 & 2.08469700 & 1.68526300 & -0.55621700 \\
\hline $\mathbf{N}$ & -2.96712900 & -2.33380300 & 0.00617900 \\
\hline $\mathbf{N}$ & 4.10219600 & -0.46000700 & 0.07383900 \\
\hline $\mathbf{N}$ & -1.56527800 & 3.16363900 & -0.09695100 \\
\hline C & -0.14151200 & 1.15603000 & 0.00488200 \\
\hline C & -1.64560300 & -2.30776800 & 0.09437100 \\
\hline $\mathrm{H}$ & -1.18680000 & -3.29142400 & 0.16098400 \\
\hline C & 1.64819900 & -0.58223900 & -0.12294900 \\
\hline C & -1.08968800 & 0.12065500 & 0.40021600 \\
\hline C & -0.75696300 & -1.25350800 & 0.03965500 \\
\hline C & -0.41649900 & 2.50658500 & -0.00874800 \\
\hline $\mathrm{H}$ & 0.45930800 & 3.15072600 & -0.00512200 \\
\hline C & 0.63907300 & -1.64257000 & -0.16122200 \\
\hline C & 2.95119300 & -1.03701900 & -0.22472100 \\
\hline $\mathrm{H}$ & 3.04841300 & -2.06890700 & -0.55414600 \\
\hline C & 1.26686600 & 0.81829600 & -0.22818400 \\
\hline C & -2.82086600 & 2.55459400 & -0.47929500 \\
\hline $\mathrm{H}$ & -2.62789200 & 1.72181300 & -1.15467900 \\
\hline $\mathrm{H}$ & -3.41702500 & 3.30313500 & -1.00039700 \\
\hline $\mathrm{H}$ & -3.37091100 & 2.17134000 & 0.37903000 \\
\hline C & -3.68915400 & -3.56605100 & 0.25436100 \\
\hline $\mathrm{H}$ & -2.99161000 & -4.36096100 & 0.50441600 \\
\hline $\mathrm{H}$ & -4.26398200 & -3.85371000 & -0.62781300 \\
\hline $\mathrm{H}$ & -4.38305800 & -3.43804100 & 1.08708200 \\
\hline C & -3.77527800 & -1.21669300 & -0.43264100 \\
\hline $\mathrm{H}$ & -4.08723100 & -0.58453300 & 0.39761800 \\
\hline $\mathrm{H}$ & -4.65003600 & -1.61225300 & -0.94818600 \\
\hline $\mathrm{H}$ & -3.20098700 & -0.60313900 & -1.12558100 \\
\hline C & 4.23972800 & 0.74175600 & 0.87109400 \\
\hline $\mathrm{H}$ & 4.34990700 & 1.63109200 & 0.25389800 \\
\hline $\mathrm{H}$ & 5.11977800 & 0.62278100 & 1.50421200 \\
\hline H & 3.36165400 & 0.86884900 & 1.49820800 \\
\hline
\end{tabular}




$\begin{array}{lrrr}\mathrm{C} & -1.59574000 & 4.60164900 & 0.07982900 \\ \mathrm{H} & -2.23435200 & 4.86846500 & 0.92398900 \\ \mathrm{H} & -1.99178300 & 5.08584700 & -0.81477200 \\ \mathrm{H} & -0.59317600 & 4.97365800 & 0.27454800 \\ \mathrm{C} & 5.35110700 & -1.10681800 & -0.27973400 \\ \mathrm{H} & 5.93228200 & -1.32620400 & 0.61781300 \\ \mathrm{H} & 5.94508000 & -0.45203600 & -0.91956200 \\ \mathrm{H} & 5.15312700 & -2.03038800 & -0.81699900\end{array}$

Calculated Energy-Optimized Atomic Coordinates for S2

$\begin{array}{lrrr}\text { O } & 2.35895800 & 0.68476800 & 0.19308800 \\ \text { O } & -0.64663900 & -2.89716000 & 0.39656600 \\ \text { O } & -2.11920600 & 1.29189500 & -1.02013700 \\ \text { N } & 3.19332500 & -2.16627500 & -0.12748900 \\ \text { N } & -3.98598400 & -0.80857700 & 0.09753900 \\ \text { N } & 1.04323500 & 3.40609600 & 0.14915400 \\ \mathrm{C} & 0.07732100 & 1.14407600 & -0.15410400 \\ \mathrm{C} & 1.91578200 & -2.14828000 & 0.21362900 \\ \mathrm{H} & 1.56351800 & -3.08754600 & 0.63470600 \\ \mathrm{C} & -1.52535300 & -0.76031900 & -0.04692200 \\ \mathrm{C} & 1.20894400 & 0.25250700 & 0.06013100 \\ \mathrm{C} & 0.93600600 & -1.17930500 & 0.07866100 \\ \mathrm{C} & 0.17559700 & 2.51389200 & -0.30494800 \\ \mathrm{H} & -0.64223500 & 2.95427400 & -0.87172300 \\ \mathrm{C} & -0.42771900 & -1.69947100 & 0.17380400 \\ \mathrm{C} & -2.78203400 & -1.33888900 & -0.03749900 \\ \mathrm{H} & -2.78339000 & -2.42490500 & -0.09282700 \\ \mathrm{C} & -1.25891300 & 0.61025100 & -0.45349700 \\ \mathrm{C} & 1.97421600 & 3.19205300 & 1.23856800 \\ \mathrm{H} & 1.68925200 & 2.31270700 & 1.80488900 \\ \mathrm{H} & 1.93593900 & 4.07164800 & 1.88485400 \\ \mathrm{H} & 2.99395700 & 3.05733400 & 0.88143500 \\ \mathrm{C} & 4.04128000 & -3.26091900 & 0.30326800 \\ \mathrm{H} & 3.48115200 & -3.93687200 & 0.94363800 \\ \mathrm{H} & 4.89397500 & -2.87524100 & 0.86480500 \\ \mathrm{H} & 4.42152400 & -3.80950600 & -0.56055400 \\ \mathrm{C} & 3.81996100 & -1.24521800 & -1.05445800 \\ \mathrm{H} & 4.48560300 & -1.82448200 & -1.69720700 \\ \mathrm{H} & 4.39283800 & -0.47613100 & -0.53996800 \\ \mathrm{H} & 3.06385100 & -0.75941300 & -1.66258600 \\ \mathrm{C} & -4.24051900 & 0.54015300 & 0.55843300 \\ \mathrm{H} & -5.16045900 & 0.52746700 & 1.14323800 \\ \mathrm{H} & -4.33577200 & 1.24300800 & -0.26706000 \\ \mathrm{H} & -3.41975000 & 0.87080200 & 1.19102300 \\ \mathrm{H} & 0.98622900 & 4.77352400 & -0.32904700 \\ & 1.94840200 & 5.06120600 & -0.75703800\end{array}$




$\begin{array}{lrrr}\text { H } & 0.76229700 & 5.45608700 & 0.49348300 \\ \mathrm{H} & 0.22374300 & 4.87114700 & -1.09715000 \\ \mathrm{C} & -5.16599200 & -1.62511700 & -0.11026900 \\ \mathrm{H} & -5.76974800 & -1.21551600 & -0.92198300 \\ \mathrm{H} & -5.77728300 & -1.64555800 & 0.79361000 \\ \mathrm{H} & -4.87519800 & -2.63886000 & -0.37264900\end{array}$

Calculated Energy-Optimized Atomic Coordinates for S3

\begin{tabular}{|c|c|c|c|}
\hline 0 & -2.28229000 & 0.41776700 & 0.86819900 \\
\hline 0 & 0.95282300 & -2.84225200 & 0.26820100 \\
\hline 0 & 2.13562400 & 1.67570800 & 0.50992500 \\
\hline $\mathbf{N}$ & -2.95783800 & -2.33707600 & -0.19505900 \\
\hline $\mathbf{N}$ & 4.06865000 & -0.49057500 & -0.25115700 \\
\hline $\mathbf{N}$ & -1.50199800 & 3.19625800 & -0.04185700 \\
\hline C & -0.14914800 & 1.16023500 & 0.23825000 \\
\hline C & -1.67569500 & -2.30363000 & 0.1371880 \\
\hline $\mathrm{H}$ & -1.24496300 & -3.28115900 & $0.3389840 \mathrm{C}$ \\
\hline C & 1.63369100 & -0.59010600 & 0.15947100 \\
\hline $\mathrm{C}$ & -1.16888600 & 0.13440200 & 0.41200700 \\
\hline C & -0.78294900 & -1.25279900 & 0.18926000 \\
\hline C & -0.40857000 & 2.51415600 & 0.2686240 \\
\hline $\mathrm{H}$ & 0.44434500 & 3.13767800 & 0.52508700 \\
\hline C & 0.62599600 & -1.65050200 & 0.2223300 \\
\hline C & 2.93858700 & -1.05597400 & 0.1346670 \\
\hline $\mathrm{H}$ & 3.05221500 & -2.09426400 & 0.43725000 \\
\hline C & 1.27741900 & 0.81264300 & 0.2942620 \\
\hline C & -2.63909600 & 2.63617300 & -0.74008800 \\
\hline $\mathrm{H}$ & -2.30323400 & 1.83651400 & -1.39988800 \\
\hline $\mathrm{H}$ & -3.09132900 & 3. 42535800 & -1.3402240 \\
\hline H & -3.37605900 & 2.21834400 & -0.0560100 \\
\hline C & -3.71920200 & -3.55941300 & -0.0279580 \\
\hline $\mathrm{H}$ & -3.08752800 & -4.33435500 & 0.3982320 \\
\hline $\mathrm{H}$ & -4.11002500 & -3.89964200 & -0.98874600 \\
\hline $\mathrm{H}$ & -4.56252900 & -3.39084700 & 0.64398100 \\
\hline C & -3.65791100 & -1.25129000 & -0.84798000 \\
\hline H & -4.10230200 & -0.55987900 & -0.1335900 \\
\hline $\mathrm{H}$ & -4.43094200 & -1.68069800 & -1.4846810 \\
\hline $\mathrm{H}$ & -2.95964900 & -0.68897600 & -1.4672190 \\
\hline C & 4.16229400 & 0.71454200 & -1.04970500 \\
\hline H & 4.98862700 & 0.58587500 & -1.75001300 \\
\hline $\mathrm{H}$ & 4.33499500 & 1.59738800 & -0.4378460 \\
\hline $\mathrm{H}$ & 3.24136100 & 0.85827200 & -1.6073180 \\
\hline C & -1.56145400 & 4.62375700 & 0.2021420 \\
\hline $\mathrm{H}$ & -2.38108200 & 4.85860700 & 0.8836330 \\
\hline $\mathrm{H}$ & -1.72880900 & 5.16243100 & -0.73250500 \\
\hline $\mathrm{H}$ & -0.63154900 & 4.96318700 & $0.650844 \mathrm{C}$ \\
\hline
\end{tabular}




$\begin{array}{lrrr}\text { C } & 5.33313500 & -1.15515300 & 0.00120100 \\ \text { H } & 5.97798900 & -0.51655400 & 0.60742000 \\ \text { H } & 5.84555600 & -1.36366100 & -0.93965100 \\ \text { H } & 5.16469200 & -2.08591100 & 0.53614700\end{array}$

Calculated Energy-Optimized Atomic Coordinates for S4

\begin{tabular}{|c|c|c|c|}
\hline 0 & -2.13960602 & -1.51538219 & 0.64586297 \\
\hline 0 & -0.29222219 & 2.71353012 & -0.10720392 \\
\hline 0 & 2.20766236 & -1.08355250 & -1.03859579 \\
\hline $\mathbf{N}$ & -3.78981730 & 0.88806454 & -0.10465950 \\
\hline $\mathbf{N}$ & 2.70910186 & 2.72649623 & 0.05625173 \\
\hline $\mathrm{N}$ & 1.14782424 & -3.65002885 & 0.19543529 \\
\hline C & 0.11637639 & -1.41533357 & -0.01816683 \\
\hline C & -2.54589901 & 1.28559119 & 0.11134125 \\
\hline H & -2.45884601 & 2.34653117 & 0.33350657 \\
\hline C & 1.13163279 & 0.84953042 & -0.20394958 \\
\hline C & -1.20038532 & -0.82436910 & 0.23468675 \\
\hline C & -1.34203582 & 0.61009062 & 0.03993308 \\
\hline C & 0.14539730 & -2.79423713 & 0.06916535 \\
\hline $\mathrm{H}$ & -0.83436147 & -3.26596887 & 0.09031803 \\
\hline C & -0.16679817 & 1.48463190 & -0.04768896 \\
\hline C & 2.31632406 & 1.53851526 & -0.37597494 \\
\hline $\mathrm{H}$ & 3.08581331 & 0.99082193 & -0.91531872 \\
\hline C & 1.22855268 & -0.59323739 & -0.46855828 \\
\hline C & 2.49800395 & -3.29009604 & 0.57473999 \\
\hline $\mathrm{H}$ & 2.48668234 & -2.36922837 & 1.15219023 \\
\hline $\mathrm{H}$ & 3.14043550 & -3.14119083 & -0.29122841 \\
\hline $\mathrm{H}$ & 2.89661435 & -4.09430403 & 1.19387060 \\
\hline C & -4.89700022 & 1.76536860 & 0.22096157 \\
\hline $\mathrm{H}$ & -4.52464272 & 2.68927700 & 0.65566183 \\
\hline $\mathrm{H}$ & -5.55975171 & 1.28392732 & 0.94226228 \\
\hline $\mathrm{H}$ & -5.47700453 & 1.99427946 & -0.67512622 \\
\hline C & -4.16016927 & -0.33778130 & -0.78170680 \\
\hline $\mathrm{H}$ & -5.03038417 & -0.12479294 & -1.40408700 \\
\hline $\mathrm{H}$ & -4.39743525 & -1.13583185 & -0.08070057 \\
\hline $\mathrm{H}$ & -3.34130869 & -0.67255745 & -1.41264979 \\
\hline C & 2.04668134 & 3.48680721 & 1.09691903 \\
\hline $\mathrm{H}$ & 1.37793173 & 4.24229471 & 0.68975473 \\
\hline $\mathrm{H}$ & 2.81760525 & 3.96618646 & 1.70204149 \\
\hline $\mathrm{H}$ & 1.46158347 & 2.81981900 & 1.72460293 \\
\hline C & 0.90441285 & -5.07360197 & 0.06690131 \\
\hline $\mathrm{H}$ & 1.18069715 & -5.59102942 & 0.98748509 \\
\hline $\mathrm{H}$ & 1.50078550 & -5.48644071 & -0.74881744 \\
\hline $\mathrm{H}$ & -0.14584932 & -5.25386752 & -0.14679379 \\
\hline C & 3.96891508 & 3.28163968 & -0.39803559 \\
\hline $\mathrm{H}$ & 4.65191637 & 3.41514628 & 0.44316564 \\
\hline
\end{tabular}

S14 


\section{References}

(1) Lawrence, A. L.; Adlington, R. M.; Baldwin, J. E.; Lee, V.; Kershaw, J. A.; Thompson, A. L. Org. Lett. 2010, 12, 1676.

(2) Sheldrick, G. M. Acta Crystallogr. Sect. A Found. Adv. 2015, 71, 3.

(3) Bax, A.; Ikura, M.; Kay, L. E.; Torchia, D. A.; Tschudin, R. J. Magn. Reson. 1990, 86, 304.

(4) Norwood, T. J.; Boyd, J.; Heritage, J. E.; Soffe, N.; Campbell, I. D. J. Magn. Reson. 1990, $87,488$.

(5) Levitt, M. H. Spin Dynamics: Basics of Nuclear Magnetic Resonance, 2nd ed.; John Wiley \& Sons, 2008.

(6) Frisch, M. J.; Trucks, G. W.; Schlegel, H. B.; Scuseria, G. E.; Robb, M. A.; Cheeseman, J. R.; Scalmani, G.; Barone, V.; Mennucci, B.; Petersson, G. A.; Nakatsuji, H.; Caricato, M.; Li, X.; Hratchian, H. P.; Izmaylov, A. F.; Bloino, J.; Zheng, G.; Sonnenberg, J. L.; Hada, M.; Ehara, M.; Toyota, K.; Fukuda, R.; Hasegawa, J.; Ishida, M.; Nakajima, T.; Honda, Y.; Kitao, O.; Nakai, H.; Vreven, T.; Montgomery Jr., J. A.; Peralta, J. E.; Ogliaro, F.; Bearpark, M.; Heyd, J. J.; Brothers, E.; Kudin, K. N.; Staroverov, V. N.; Kobayashi, R.; Normand, J.; Raghavachari, K.; Rendell, A.; Burant, J. C.; Iyengar, S. S.; Tomasi, J.; Cossi, M.; Rega, N.; Millam, J. M.; Klene, M.; Knox, J. E.; Cross, J. B.; Bakken, V.; Adamo, C.; Jaramillo, J.; Gomperts, R.; Stratmann, R. E.; Yazyev, O.; Austin, A. J.; Cammi, R.; Pomelli, C.; Ochterski, J. W.; Martin, R. L.; Morokuma, K.; Zakrzewski, V. G.; Voth, G. A.; Salvador, P.; Dannenberg, J. J.; Dapprich, S.; Daniels, A. D.; Farkas, Ö.; Foresman, J. B.; Ortiz, J. V; Cioslowski, J.; Fox, D. J. Gaussian Inc.: Wallingford, CT 2009 ,.

(7) Zhao, Y.; Truhlar, D. G. Theor. Chem. Acc. 2008, 120, 215.

(8) Dunning, T. H. J. Chem. Phys. 1989, 90, 1007.

(9) Kendall, R. A.; Dunning, T. H.; Harrison, R. J. J. Chem. Phys. 1992, 96, 6796.

(10) Davidson, E. R. Chem. Phys. Lett. 1996, 260, 514.

(11) Tomasi, J.; Mennucci, B.; Cammi, R. Chem. Rev. 2005, 105, 2999.

(12) Bauernschmitt, R.; Ahlrichs, R. Chem. Phys. Lett. 1996, 256, 454.

(13) Casida, M. E.; Jamorski, C.; Casida, K. C.; Salahub, D. R. J. Chem. Phys. 1998, 108, 4439. 
(14) Stratmann, R. E.; Scuseria, G. E.; Frisch, M. J. J. Chem. Phys. 1998, 109, 8218.

(15) Furche, F.; Ahlrichs, R. J. Chem. Phys. 2002, 117, 7433. 